\title{
Comparison of the Kato-Katz, Wet Mount, and Formol-Ether Concentration Diagnostic Techniques for Intestinal Helminth Infections in Ethiopia
}

\author{
Mengistu Endris, Zinaye Tekeste, Wossenseged Lemma, and Afework Kassu \\ School of Biomedical and Laboratory Sciences, College of Medicine and Health Sciences, University of Gondar, \\ P.O. Box 196, Gondar, Ethiopia
}

Correspondence should be addressed to Zinaye Tekeste; zinzn98@yahoo.com

Received 13 July 2012; Accepted 19 September 2012

Academic Editors: A. Domingos, H. Madsen, and D. C. Simcock

Copyright (C) 2013 Mengistu Endris et al. This is an open access article distributed under the Creative Commons Attribution License, which permits unrestricted use, distribution, and reproduction in any medium, provided the original work is properly cited.

\begin{abstract}
Objective. The aim of this study was to evaluate the operational characteristics (sensitivity and negative predictive value (NPV)) of wet mount, formol-ether concentration (FEC), and Kato-Katz techniques for the determination of intestinal parasitic infections. Method. A total of 354 faecal specimens were collected from students in Northwest Ethiopia and screened with Kato-Katz, wet mount, and FEC for the presence of intestinal parasitic infection. Since a gold standard test is not available for detection of intestinal parasites, the combined results from the three methods were used as diagnostic gold standard. Result. The prevalences of intestinal parasites using the single wet mount, FEC, and Kato-Katz thick smear techniques were $38.4 \%, 57.1 \%$, and $59 \%$, respectively. Taking the combined results of three techniques as a standard test for intestinal parasitic infection, the sensitivity and negative predictive value of Kato-Katz is $81.0 \%$ (confidence interval $(\mathrm{CI})=0.793-0.810)$ and $66.2 \%(\mathrm{CI}=0.63-0.622)$, respectively. The FEC detected 56 negative samples that were positive by the gold standard, indicating $78.3 \%(\mathrm{CI}=0.766-0.783)$ and $63.2 \%(\mathrm{CI}=0.603-63)$ sensitivity and NPV, respectively. Furthermore, Kato-Katz detects 113 cases that were negative by a single wet mount. The $\kappa$ agreement between the wet mount and Kato-Katz methods for the diagnosis of Ascaris lumbricoides and hookworm was substantial $(\kappa=0.61$ for Ascaris lumbricoides, $\kappa=0.65$ for hookworm).
\end{abstract}

\section{Background}

Intestinal parasitic infections are among the most common infections worldwide. It is estimated that 3.5 billion people are affected, and 450 million are ill as a result of these infections, the majority being children [1]. In Ethiopia, intestinal parasitic infection is the second most predominant cause of outpatient morbidity. However, there are difficulties in estimating the exact burden of parasitic infections in the country [2]. Although there are several factors that make estimating the number and burden of intestinal parasitic infections difficult, lack of accurate diagnostic tools is the major one [3-9].

Although several diagnostic methods such as KatoKatz and Formol-Ether Concentration (FEC) techniques are available, direct wet mount is the commonly used as a reliable diagnosis method for the diagnosis of intestinal parasitic infections generally in Africa and particularly in Ethiopia [10-13]. However, low sensitivity of the direct wet mount technique has been reported in the detection of lowintensity infection elsewhere [14]. This shows that the use of direct wet mount as a confirmatory test will significantly increase misdiagnosis of intestinal helminth infections. The reliable diagnosis of intestinal parasitic infections requires a more rapid, easy, and sensitive method. Therefore, this study aimed to evaluate the operational characteristics (sensitivity and negative predictive value (NPV)) of wet mount, FEC, and Kato-Katz techniques in intestinal parasitic infection endemic locality of Ethiopia.

\section{Methods}

2.1. Study Design and Area. A cross-sectional study was conducted in Atse Fasil Elementary School from March 10 
TABLE 1: Wet mount, Kato-Katz, and FEC techniques results compared to the gold standard from Atse Fasil General Elementary School, Northwest Ethiopia, March 10-June 30, 2008.

\begin{tabular}{|c|c|c|c|c|c|c|c|c|}
\hline \multirow{2}{*}{ Method } & \multirow{2}{*}{ Result } & \multicolumn{2}{|c|}{ Gold standard } & \multirow{2}{*}{ Total (\%) } & \multirow[b]{2}{*}{ NPV } & \multirow{2}{*}{$95 \%$ CI } & \multirow{2}{*}{ Sensitivity } & \multirow{2}{*}{$95 \% \mathrm{CI}$} \\
\hline & & Positive (\%) & Negative (\%) & & & & & \\
\hline \multirow{2}{*}{ Wet mount } & Positive & $136(38.4)$ & 0 & $136(38.4)$ & \multirow{2}{*}{$44.0 \%$} & \multirow{2}{*}{$0.42-0.44$} & \multirow{2}{*}{$52.7 \%$} & \multirow{2}{*}{$0.51-0.53$} \\
\hline & Negative & $122(34.5)$ & $96(27.1)$ & $218(61.6)$ & & & & \\
\hline \multirow{2}{*}{ FEC } & Positive & $202(57.1)$ & 0 & $202(57.1)$ & \multirow{2}{*}{$63.2 \%$} & \multirow{2}{*}{$0.60-0.63$} & \multirow{2}{*}{$78.3 \%$} & \multirow{2}{*}{$0.76-0.78$} \\
\hline & Negative & $56(15.8)$ & $96(27.1)$ & $152(42.9)$ & & & & \\
\hline \multirow{2}{*}{ Kato-Katz } & Positive & $209(59.0)$ & 0 & $209(59.0)$ & \multirow{2}{*}{$66.2 \%$} & \multirow{2}{*}{$0.63-0.62$} & \multirow{2}{*}{$81.0 \%$} & \multirow{2}{*}{$0.79-0.81$} \\
\hline & Negative & $49(13.8)$ & $96(27.1)$ & $145(41.0)$ & & & & \\
\hline
\end{tabular}

FEC: formol-ether concentration, NPV: negative predictive value.

to June 30, 2008 in Northwest Ethiopia, latitude of 12.55 $\left(12^{\circ} 33^{\prime} 0 \mathrm{~N}\right)$, a longitude of $37.43\left(37^{\circ} 25^{\prime} 32 \mathrm{E}\right)$, and an elevation of 1,994 meters above sea level [15].

2.2. Study Population. A total of 354 students were included in the study. Students who had no history of antihelmenthic drug administration in the two weeks prior to screening, absence of any other serious chronic infection, and had ability to give stool samples were included in the study.

2.3. Stool Collection and Analysis. Students were provided with plastic stool cups and asked to bring approximately $3 \mathrm{gm}$ of fresh stool of their own. Approximately $41.7 \mathrm{mg}$ and $20 \mathrm{mg}$ of the stool specimen were used to prepare a single KatoKatz thick smear and wet mount preparation, respectively. One gram of stool sample was analyzed by FEC technique [16]. All the samples were processed in the temporally established laboratory except the Kato-Katz smear count of the common helminthes, which was done at the laboratory of the University of Gondar. The egg per gram of faeces (EPGs) counts for hookworm were done immediately in the temporary laboratory. The Kato-thick smear counts for all the common helminthes except for hookworm were done at the laboratory of University of Gondar.

2.4. Quality Control. Before starting the actual work, quality of reagents and instruments was checked by experienced laboratory technicians. The specimens were also checked for serial number, quantity, and procedures of collection.

To eliminate observer bias, each stool sample was examined immediately in the temporary laboratory by two experienced laboratory technicians. The technicians were not told about the health and other status of the study participants. In cases where the results were discordant, a third expert reader was used. The results of the third expert reader were considered the final result.

2.5. Data Analysis. Since a "gold" standard test is not available for detection of intestinal parasites, the operational characteristics (sensitivity and NPV) were estimated using the combined results from the three methods as diagnostic "gold" standard $[17,18]$.

Data were analyzed using SPSS version 16 and JavaStat software. Sensitivities, NPV, and kappa were determined for the various tests to evaluate their operational characteristics. $P$ values $<0.05$ were considered statistically significant.

2.6. Ethical Considerations. The study protocol was reviewed and approved by the Ethical Review Committee of the Department of Medical Laboratory Technology, University of Gondar. Written informed consent was obtained from all study participants and mothers/caretakers of students under 18 who participated in the study after explaining the purpose and objective of the study. Students who were positive for intestinal parasites were treated based on the recommended drug regimen at Azezo Clinic, Gondar.

\section{Result}

A total of 354 students aged 5-19 years participated in the study; 146 (53.9\%) and 125 (46.1\%) were males and females, respectively.

3.1. Prevalence. The prevalences of intestinal parasites using single wet mount, FEC, and Kato-Katz thick smear techniques were $38.4 \%, 57.1 \%$, and $59 \%$, respectively (Table 1 ). The detection rate when two techniques used at a time was $69.8 \%$ (for Kato-thick and FEC), 61\% (for wet mount and FEC), and 68\% (for wet mount and Kato-thick smear technique). The detection rate was $72.9 \%$ (258/354) when all the three techniques were used together. The overall prevalence of Schistosoma mansoni, Ascaris lumbricoides, Trichuris trichiura, and hookworm infections was $43.5 \%$, $28.8 \%, 18.1 \%$, and $8.2 \%$, respectively (Table 2 ).

3.2. Sensitivity and NPV. Among the 354 study participants diagnosed 209 were found to be positive while 145 were negative by Kato-Katz. The sensitivity and NPV of KatoKatz are $81.0 \%$ (confidence interval $(\mathrm{CI})=0.793-0.810)$ and $66.2 \%$ (CI $=0.63-0.622)$, respectively (Table 1$)$. The FEC detected 56 negative samples that were positive by the gold standard, indicating 78.3\% (CI $=0.766-0.783)$ and $63.2 \%(\mathrm{CI}$ $=0.603-63$ ) sensitivity and NPV, respectively (Table 1 ).

3.3. Agreement in Test Results. As compared to single FEC, single Kato-Katz detected 53, 83, 56, and 95 cases of Ascaris lumbricoides, Schistosoma mansoni, Trichuris trichiura, and hook worm, respectively (Table 3), indicating substantial 
TABLE 2: Wet mount, Kato-Katz, and FEC techniques results compared to the gold standard by species of common helminthes from students of Atse Fasil General Elementary School, Northwest Ethiopia, March 10-June 30, 2008.

\begin{tabular}{|c|c|c|c|c|}
\hline Parasite & Technique & Infected students no. (\%) & NPV (95\% CI) & Sensitivity $(95 \% \mathrm{CI})$ \\
\hline \multirow{4}{*}{ S. mansoni } & Gold standard & $154(43.5)$ & & \\
\hline & Kato katz & $148(41.8)$ & $97.1 \%(0.95-0.97)$ & $96.1 \%(0.94-0.96)$ \\
\hline & FEC & $90(25.4)$ & $75.8 \%(0.74-0.75)$ & $58.4 \%(0.55-0.58)$ \\
\hline & Wet mount & $34(9.6)$ & $62.5 \%(0.61-0.62)$ & $22.1 \%(0.194-0.221)$ \\
\hline \multirow{4}{*}{ A. lumbricoides } & Gold standard & $102(28.8)$ & & \\
\hline & Kato-Katz & $95(26.8)$ & $97.3 \%(0.95-0.97)$ & $93.1 \%(0.89-0.93)$ \\
\hline & FEC & $83(23.4)$ & $93.0 \%(0.92-0.93)$ & $81.4 \%(0.77-0.81)$ \\
\hline & Wet mount & $53(15)$ & $83.7 \%(0.82-0.83)$ & $52.0 \%(0.48-0.52)$ \\
\hline \multirow{4}{*}{ T. trichiura } & Gold standard & $64(18.1)$ & & \\
\hline & Kato-Katz & $58(16.4)$ & $98.0 \%(0.97-0.98)$ & $90.6 \%(0.85-0.91)$ \\
\hline & FEC & $37(10.5)$ & $91.5 \%(0.90-0.92)$ & $57.8 \%(0.52-0.58)$ \\
\hline & Wet mount & $8(2.3)$ & $83.8 \%(0.83-0.84)$ & $12.5 \%(0.07-0.12)$ \\
\hline \multirow{4}{*}{ Hook worm } & Gold standard & $29(8.2)$ & & \\
\hline & Kato-Katz & $20(5.6)$ & $97.3 \%(0.96-0.97)$ & $69.0 \%(0.57-0.69)$ \\
\hline & FEC & $21(5.9)$ & $97.6 \%(0.97-0.98)$ & $72.4 \%(0.60-0.72)$ \\
\hline & Wet mount & $11(3.1)$ & $94.8 \%(0.94-0.95)$ & $37.9 \%(0.27-0.38)$ \\
\hline
\end{tabular}

FEC: formol-ether concentration, NPV: negative predictive value.

TABLE 3: Agreement between a single Kato-Katz and FEC technique for the diagnosis of each intestinal parasite in students from Atse Fasil General Elementary School, Northwest Ethiopia, March 10-June 30, 2008.

\begin{tabular}{|c|c|c|c|c|c|}
\hline \multirow{2}{*}{ Parasite } & \multirow{2}{*}{ FEC } & \multicolumn{2}{|c|}{ Kato-Katz } & \multirow{2}{*}{ Total } & \multirow{2}{*}{$\kappa$ agreement $(P)$} \\
\hline & & Positive & Negative & & \\
\hline \multirow{3}{*}{ S. mansoni } & Positive & 85 & 5 & 85 & \multirow{2}{*}{$0.58(P<0.001)$} \\
\hline & Negative & 62 & 202 & 264 & \\
\hline & Total & 147 & 207 & 354 & \\
\hline \multirow{3}{*}{ A. lumbricoides } & Positive & 75 & 8 & 83 & \multirow{2}{*}{$0.80(P<0.001)$} \\
\hline & Negative & 18 & 253 & 271 & \\
\hline & Total & 93 & 261 & 354 & \\
\hline \multirow{3}{*}{ T. trichiura } & Positive & 31 & 6 & 37 & \multirow{2}{*}{$0.59(P<0.001)$} \\
\hline & Negative & 28 & 289 & 317 & \\
\hline & Total & 59 & 295 & 354 & \\
\hline \multirow{3}{*}{ Hook worm } & Positive & 12 & 9 & 21 & \multirow{2}{*}{$0.57(P<0.001)$} \\
\hline & Negative & 7 & 326 & 333 & \\
\hline & Total & 19 & 335 & 354 & \\
\hline
\end{tabular}

FEC: formol-ether concentration.

$(\kappa=0.80)$, moderate $(\kappa=0.58)$, and moderate $(\kappa=$ $0.59) \kappa$ agreement between the FEC and Kato-Katz for the diagnosis of Ascaris lumbricoides, Schistosoma mansoni and Trichuris trichiura, respectively (Table 3). Furthermore, KatoKatz detects 113 cases that were negative by a single wet mount (Table 4 ). The $\kappa$ agreement between the wet mount and Kato-Katz methods for the diagnosis of Ascaris lumbricoides and hook worm was substantial $(\kappa=0.61$ for Ascaris lumbricoides, $\kappa=0.65$ for hookworm) (Table 4 ).

\section{Discussion}

A single Kato-Katz had significantly lower detection capacity than the FEC method in diagnosing hookworm. This poor performance of the Kato-Katz in detecting hookworm infection is explained by the following facts. First, hookworm has lower egg laying capacity, more likely to be missed by KatoKatz. Second, hookworm eggs disappear due to glycerin when long time delays occur between Kato-Katz smear preparation and microscopic examination [19]. Furthermore, unlike FEC, small amount of fecal material is processed in Kato-Katz technique. The chance of detecting hookworm infection by Kato-Katz from small amount of faecal material is suggested to be lower $[20,21]$. Therefore, small amount of fecal material used in Kato-Katz technique may be the reason for lower detection capacity of Kato-Katz.

The finding of the present study showed that, as compared to the Kato-Katz and FEC techniques, direct wet mount 
TABLE 4: Agreement between a single Kato-Katz and wet mount for the diagnosis of each intestinal parasite in students from Atse Fasil General Elementary School, Northwest Ethiopia, March 10-June 30, 2008.

\begin{tabular}{|c|c|c|c|c|c|}
\hline \multirow{2}{*}{ Parasite } & \multirow{2}{*}{ Wet mount } & \multicolumn{2}{|c|}{ Kato-Katz } & \multirow{2}{*}{ Total } & \multirow{2}{*}{$\kappa$ agreement $(P)$} \\
\hline & & Positive & Negative & & \\
\hline \multirow{3}{*}{ S. mansoni } & Positive & 33 & 1 & 34 & \multirow{2}{*}{$0.25(P<0.001)$} \\
\hline & Negative & 114 & 206 & 320 & \\
\hline & Total & 147 & 207 & 354 & \\
\hline \multirow{3}{*}{ A. lumbricoides } & Positive & 50 & 3 & 53 & \multirow{2}{*}{$0.61(P<0.001)$} \\
\hline & Negative & 43 & 258 & 301 & \\
\hline & Total & 93 & 261 & 354 & \\
\hline \multirow{3}{*}{ T. trichiura } & Positive & 8 & 0 & 8 & \multirow{2}{*}{$0.21(P<0.001)$} \\
\hline & Negative & 51 & 295 & 346 & \\
\hline & Total & 59 & 295 & 354 & \\
\hline \multirow{3}{*}{ Hook worm } & Positive & 10 & 1 & 11 & \multirow{2}{*}{$0.65(P<0.001)$} \\
\hline & Negative & 9 & 334 & 343 & \\
\hline & Total & 19 & 335 & 354 & \\
\hline
\end{tabular}

exhibited very low sensitivity for the detection of Ascaris lumbricoides, Schistosoma mansoni, Trichuris trichiura and hookworm. The use of direct wet mount alone as an indicator of intestinal parasitic infections is also suggested to be insufficient by other studies [22]. However, in most laboratories in Ethiopia, the direct wet mount is the preferred stool parasitological detection technique. This shows that, since the use of direct wet mount as a confirmatory test will significantly increase misdiagnosis of intestinal helminth infections, the use of another diagnosing method is mandatory to decrease the consequences caused in the community due to intestinal helminth infections.

As compared to FEC and direct wet mount techniques, the Kato-Katz exhibited high sensitivity for the detection of Schistosoma mansoni, Trichuris trichiura, and Ascaris lumbricoides. This shows that the use of the Kato-Katz as a confirmatory test for Schistosoma mansoni, Trichuris trichiura, and Ascaris lumbricoides will reduce the morbidity and mortality caused by these parasites by reducing misdiagnosis. However, lack of previous similar study makes difficulty in making rigorous discussion on this finding.

In conclusion, the present study revealed that Kato-Katz technique and FEC methods showed a better sensitivity than the traditional direct wet mount method. Therefore, the employment of FEC technique as a confirmatory test in routine laboratory examination of stool and Kato-Katz in epidemiological studies will significantly aid in accurate determination and management of parasitic infections in the community.

\section{Conflict of Interests}

The authors declare that they have no conflict of interests.

\section{Authors' Contribution}

M. Endris was involved in all aspects of the project, study design, data collection, analysis, and in writing of the paper.
Z. Tekeste was involved in data analysis, interpretation, and in writing of the paper. A. Kassu and W. Lemma have made a contribution in supervision and in critically revising the paper. All authors read and approved the manuscript.

\section{Acknowledgments}

The study was financially supported by College of Medicine and Health Sciences, University of Gondar. The authors thank the study participants for their cooperation and the health management and working staff of Gondar District Health Office and University of Gondar.

\section{References}

[1] World Health Organization (WHO), Control of Tropical Diseases, WHO, Geneva, Switzerland, 1998.

[2] T. M. Tesfa-Yohannes and H. Kloos, "Intestinal parasitism," in Ecology of Health and Disease in Ethiopia, A. Z. Zein and H. Kloos, Eds., pp. 214-230, Ministry of Health, Addis Ababa, Ethiopia, 1988.

[3] M. Booth, P. Vounatsou, E. K. N'goran, M. Tanner, and J. Utzinger, "The influence of sampling effort and the performance of the Kato-Katz technique in diagnosing Schistosoma mansoni and hookworm co-infections in rural Côte d'Ivoire," Parasitology, vol. 127, no. 6, pp. 525-531, 2003.

[4] J. Utzinger, E. K. N’Goran, C. R. Caffrey, and J. Keiser, "From innovation to application: social-ecological context, diagnostics, drugs and integrated control of schistosomiasis," Acta Tropica, vol. 120, no. 1, pp. 121-137, 2010.

[5] A. Montresor, T. W. Gyorkos, D. W. Crompton, D. Bundy, and L. Savioli :, Monitoring Helminth Control Programmes: Guidelines for Monitoring the Impact of Control Programmes Aimed at Reducing Morbidity Caused by Soil-Transmitted Helminthes and Schistosomes, with Particular Reference to School-age Children, WHO, Geneva, Switzerland, 1998.

[6] WHO, "Prevention and control of schistosomiasis and soiltransmitted helminthiasis; report of a WHO expert committee," WHO Technical Report Series 912, 2002. 
[7] N. Berhe, G. Medhin, B. Erko et al., "Variations in helminth faecal egg counts in Kato-Katz thick smears and their implications in assessing infection status with Schistosoma mansoni," Acta Tropica, vol. 92, no. 3, pp. 205-212, 2004.

[8] B. Speich, S. Knopp, K. A. Mohammed et al., "Comparative cost assessment of the Kato-Katz and FLOTAC techniques for soiltransmitted helminth diagnosis in epidemiological surveys," Parasites and Vectors, vol. 3, no. 1, article 71, 2010.

[9] S. Knopp, A. F. Mgeni, I. S. Khamis et al., "Diagnosis of soiltransmitted helminths in the era of preventive chemotherapy: effect of multiple stool sampling and use of different diagnostic techniques," PLoS Neglected Tropical Diseases, vol. 2, no. 11, article e331, 2008.

[10] J. Utzinger, L. Rinaldi, L. K. Lohourignon et al., "FLOTAC: a new sensitive technique for the diagnosis of hookworm infections in humans," Transactions of the Royal Society of Tropical Medicine and Hygiene, vol. 102, no. 1, pp. 84-90, 2008.

[11] H. Kassahun, D. Abrham, Y. Yemane, and E. Birhanu, "Comparision of the Kato katz and FLOTAC techniques for the diagnosis of soil transmitted helminth infections," Parasitology International, vol. 60, no. 4, pp. 398-402, 2011.

[12] S. Knopp, L. Rinaldi, I. S. Khamis et al., "A single FLOTAC is more sensitive than triplicate Kato-Katz for the diagnosis of low-intensity soil-transmitted helminth infections," Transactions of the Royal Society of Tropical Medicine and Hygiene, vol. 103, no. 4, pp. 347-354, 2009.

[13] M. R. Tarafder, H. Carabin, L. Joseph, E. Balolong, R. Olveda, and S. T. McGarvey, "Estimating the sensitivity and specificity of Kato-Katz stool examination technique for detection of hookworms, Ascaris lumbricoides and Trichuris trichiura infections in humans in the absence of a 'gold standard"' International Journal for Parasitology, vol. 40, no. 4, pp. 399-404, 2010.

[14] B. Levecke, N. De Wilde, E. Vandenhoute, and J. Vercruysse, "Field validity and feasibility of four techniques for the detection of Trichuris in Simians: a model for monitoring drug efficacy in public health?" PLoS Neglected Tropical Diseases, vol. 3, no. 1, article e366, 2009.

[15] Gondar, Ethiopia, 2012, http://en.wikipedia.org/wiki/Gondar.

[16] WHO, Bench Aids for the Diagnosis of Intestinal Parasites, WHO, Geneva, Switzerland, 1994.

[17] L. Joseph, T. W. Gyorkos, and L. Coupal, "Bayesian estimation of disease prevalence and the parameters of diagnostic tests in the absence of a gold standard," American Journal of Epidemiology, vol. 141, no. 3, pp. 263-272, 1995.

[18] N. Dendukuri, E. Rahme, P. Bélisle, and L. Joseph, "Bayesian sample size determination for prevalence and diagnostic test studies in the absence of a gold standard test," Biometrics, vol. 60, no. 2, pp. 388-397, 2004.

[19] R. J. Dacombe, A. C. Crampin, S. Floyd et al., "Time delays between patient and laboratory selectively affect accuracy of helminth diagnosis," Transactions of the Royal Society of Tropical Medicine and Hygiene, vol. 101, no. 2, pp. 140-145, 2007.

[20] A. Hall, "Intestinal helminths of man: the interpretation of egg counts," Parasitology, vol. 85, no. 3, pp. 605-613, 1982.

[21] D. D. Lin, J. X. Liu, Y. M. Liu et al., "Routine Kato-Katz technique underestimates the prevalence of Schistosoma japonicum: a case study in an endemic area of the People's Republic of China," Parasitology International, vol. 57, no. 3, pp. 281-286, 2008.

[22] K. D. Paraemeshwarappa, C. Chandrakanth, and B. Sunil, "The prevalence of intestinal parasitic infections and the evaluation of different concentration techniques of the stool examination," Journal of Clinical and Diagnostic Research, vol. 6, no. 7, pp. 1188-1191, 2012. 

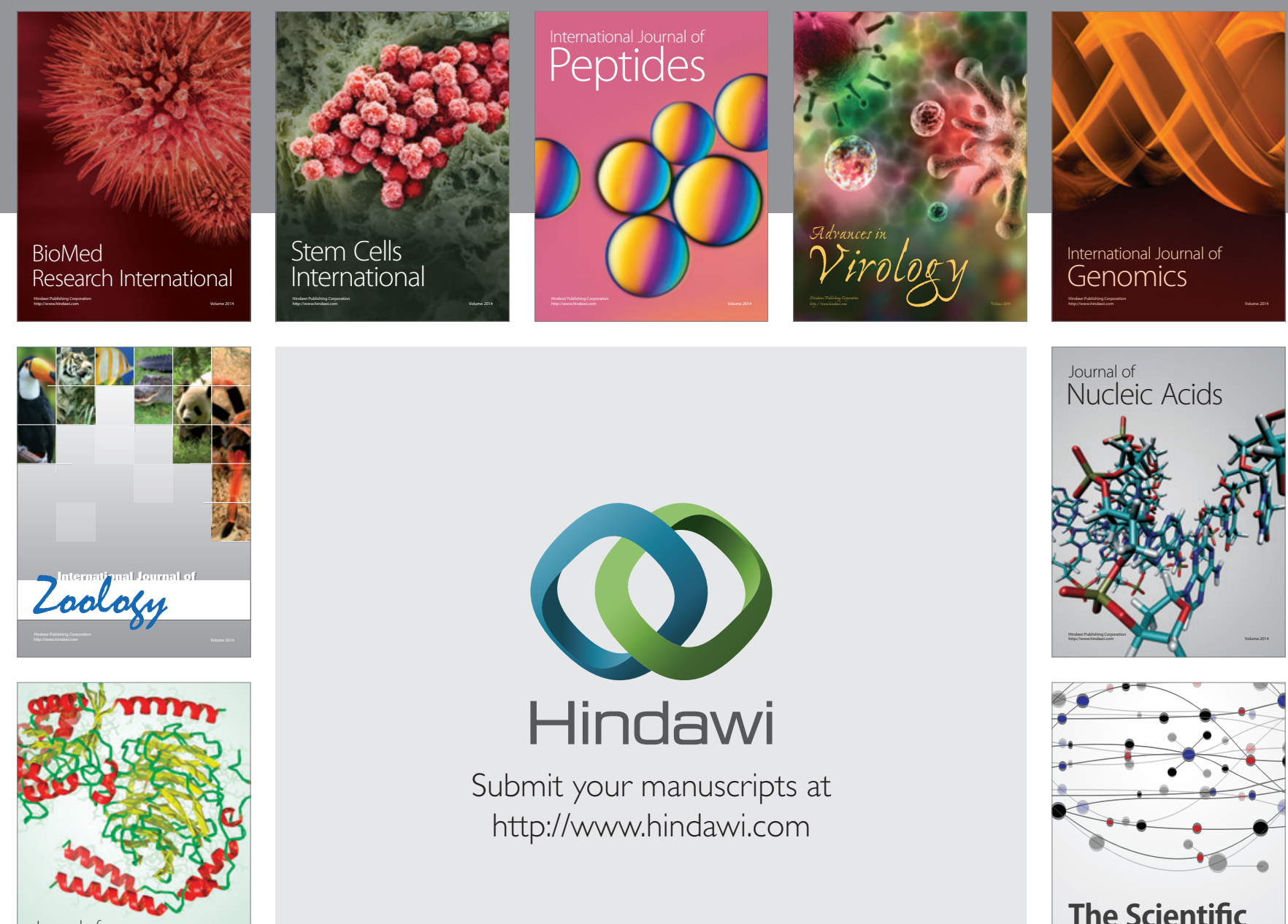

Submit your manuscripts at

http://www.hindawi.com

Journal of
Signal Transduction
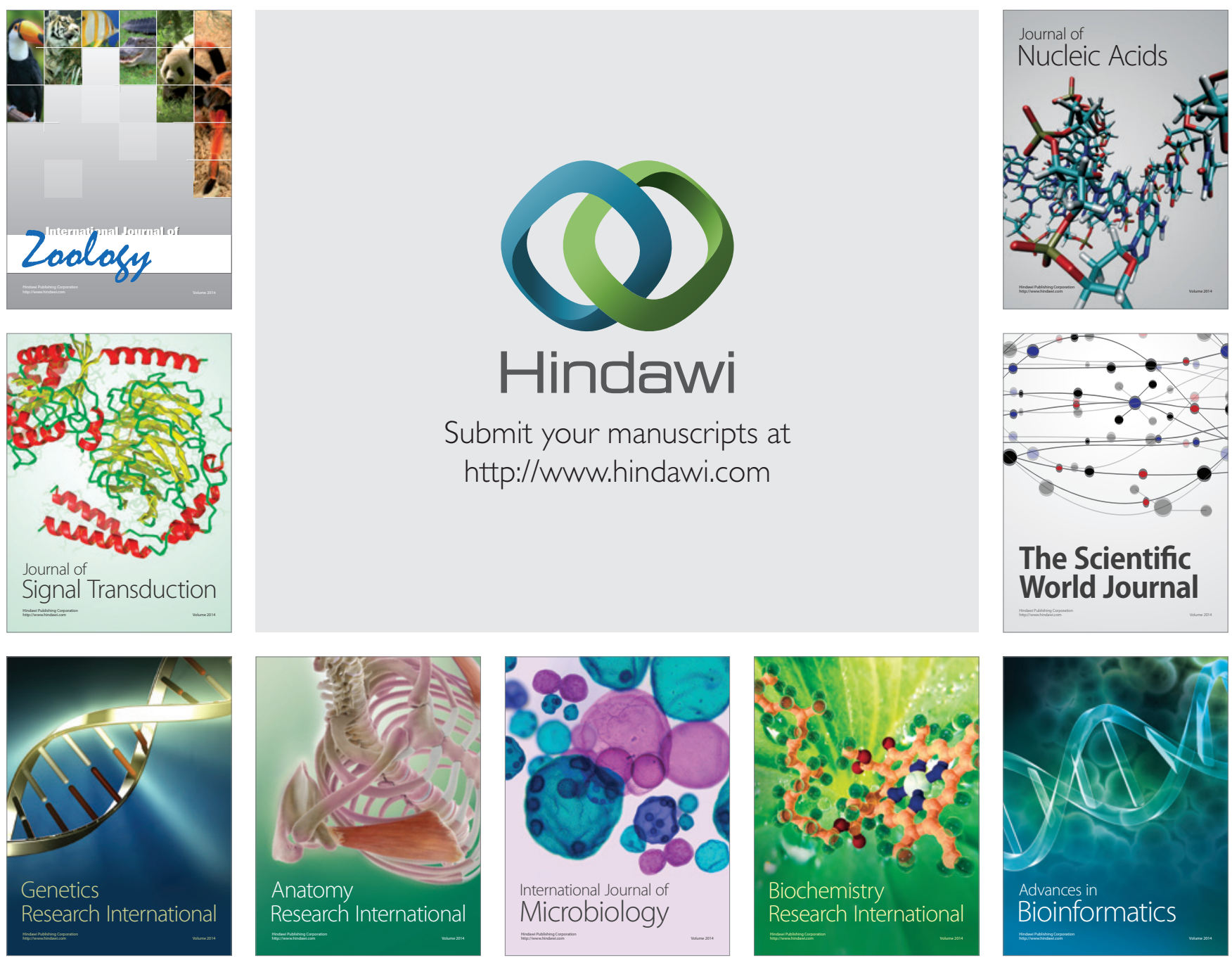

The Scientific World Journal
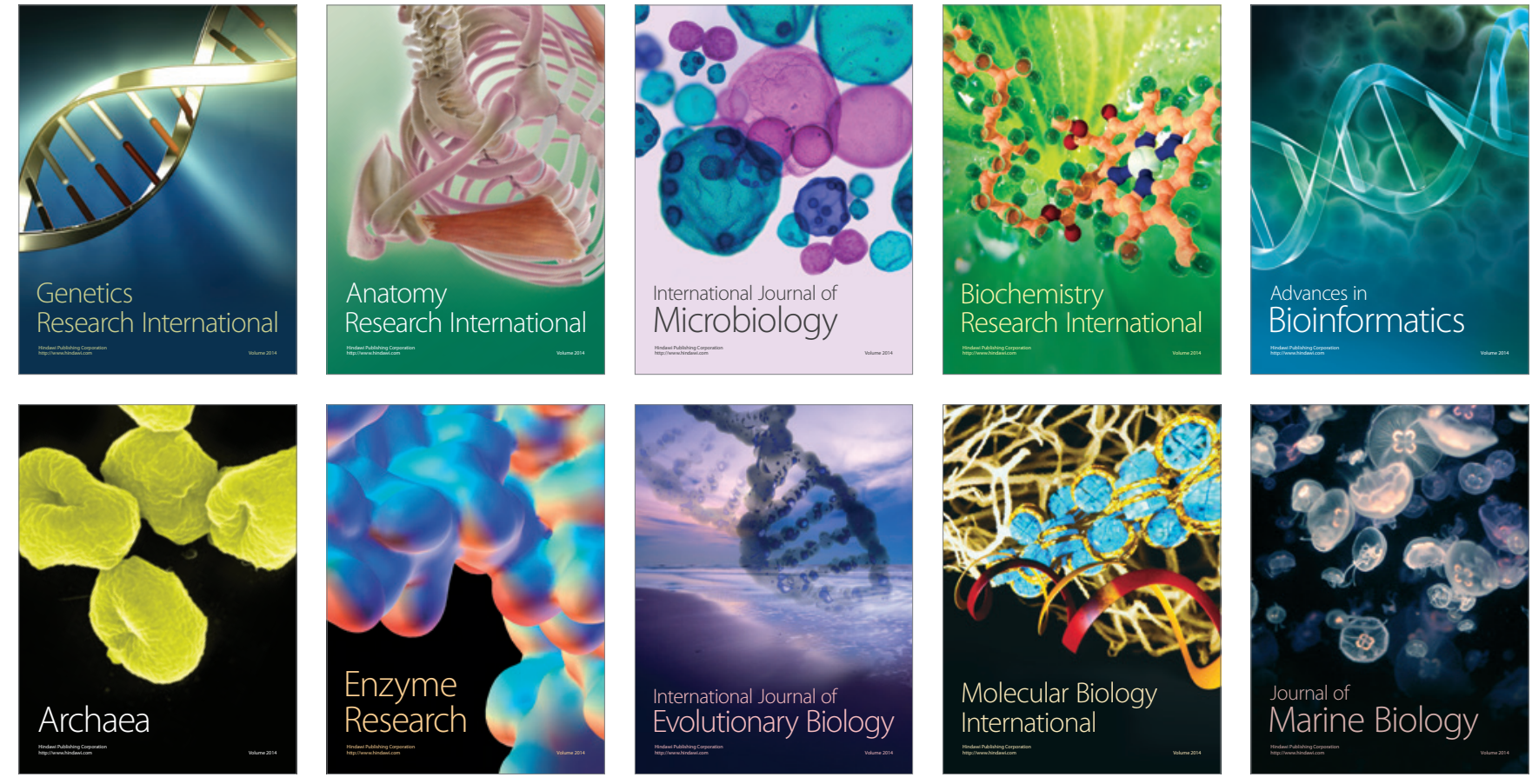